\title{
La réforme des cultes au Grand-duché du Luxembourg en 2015
}

Francis Messner

\section{CpenEdition}

\section{Journals}

Édition électronique

URL : http://journals.openedition.org/rdr/1081

DOI : $10.4000 /$ rdr. 1081

ISSN : 2534-7462

\section{Éditeur}

Presses universitaires de Strasbourg

Édition imprimée

Date de publication : 10 mai 2016

Pagination : 161-166

ISBN : 978-2-86820-954-2

ISSN : 2493-8637

\section{Référence électronique}

Francis Messner, «La réforme des cultes au Grand-duché du Luxembourg en 2015 », Revue du droit des religions [En ligne], 1 | 2016, mis en ligne le 12 février 2020, consulté le 20 novembre 2020. URL http://journals.openedition.org/rdr/1081 ; DOI : https://doi.org/10.4000/rdr.1081

\section{(a) (1) $(9)$}

La revue du droit des religions est mise à disposition selon les termes de la Creative Commons Attribution - Pas d'Utilisation Commerciale 4.0 International - CC BY-NC 4.0. 


\section{LA RÉFORME DES CULTES AU GRAND-DUCHÉ DU LUXEMBOURG EN 2015}

\section{Francis MESSNER}

Université de Strasbourg / CNRS, Droit, Religion, Entreprise et Société (DRES)

T e régime des cultes instauré au début du Xix siècle dans l'État du

LGrand-duché de Luxembourg n'a pas, malgré plusieurs aménagements successifs, échappé aux critiques soulignant son décalage avec le contexte socio-religieux contemporain. Aussi, un groupe d'experts a été chargé en 2012 par le ministre d'État de faire des propositions en vue d'une éventuelle restructuration de ce régime. Suite aux élections législatives de 2013, le nouveau gouvernement a mis en œuvre une réforme accentuant la séparation entre l'État et les religions.

La Constitution du Luxembourg du 17 octobre 1868 prévoit que les traitements et pensions des ministres des cultes sont à la charge de l'État (art. 106) et détermine par ailleurs dans son article 22 que « les rapports de l'Église avec l'État font l'objet de conventions ». Cette disposition s'appliquait, lors de la promulgation de la Constitution, au droit concordataire de l'Église catholique. Il a été étendu aux cultes les plus représentatifs à partir des années 1980.

Partie intégrante d'un département français au début du XIX ${ }^{e}$ siècle ${ }^{1}$, le Luxembourg est soumis au droit français des cultes alors en vigueur ${ }^{2}$. Le

1. Le département des Forêts.

2. Pour une histoire des relations État/Religions au Luxembourg, V. PAuly A., « State and Church in Luxemburg ", in RobBERs G. (ed.), State and Church in the European Union, Baden-Baden, Nomos, $2^{\text {nd }}$ éd. 2005, p. 305-322. 
culte catholique et la confession israélite ont été " reconnus » en application respectivement de la loi du 18 germinal an X (Convention de messidor et articles organiques du culte catholique) et du décret du 17 mars 1808. La reconnaissance du culte protestant est intervenue en 1894 conformément aux articles organiques des cultes protestants de la loi du 18 germinal an X. À partir des années 1980, la procédure de "reconnaissance " par le biais du droit unilatéral est abandonnée au profit du droit conventionnel. Le 15 juin 1982 est ainsi conclue une convention entre l'État et l'Église protestante réformée du Luxembourg, constituée d'immigrés du pays de Nassau souhaitant conserver leur indépendance par rapport aux protestants reconnus en 1894. La loi du 23 novembre 1982 portant approbation de la convention confère la personnalité de droit public à cette Église et fixe la rémunération du personnel des cultes $^{3}$. Plus d'une décennie plus tard, le 31 octobre 1997, cinq autres conventions sont conclues, dont deux avec l'archevêché de Luxembourg ${ }^{4}$, une avec la communauté israélite, une avec l'Église protestante du Luxembourg reconnue en 1894 et une avec l'Église orthodoxe hellénique ${ }^{5}$. La signature de ces cinq conventions, suivie de leur adoption par le Parlement en 1998, a transformé le système luxembourgeois des relations État-religions. Les conventions de droit public interne remplacent désormais d'une part le droit unilatéral de reconnaissance des cultes et, d'autre part, le système concordataire catholique relevant du droit public international. Tous les cultes, y compris l'Église catholique, sont traités de manière indifférenciée. Ce processus a été conforté par la signature le 27 janvier 2003 d'une convention avec l'Église anglicane

3. PAuly A., Les cultes au Luxembourg. Un modèle concordataire, Luxembourg, Forum, 1989. Messner F., "Les évolutions du statut des cultes au Luxembourg », in BAubérot J. (dir.), Religions et laïcité dans l'Europe des Douze, Paris, Syros, 1994, p. 95-105. - « Les cultes reconnus en Europe : l'exemple du Luxembourg », Revue du droit local, juin 1994.

4. L. 10 juill. 1998 modifiée portant approbation de la Convention du 31 octobre 1997 entre le Gouvernement, d'une part, et l'Archevêché, d'autre part, concernant l'organisation de l'enseignement religieux dans l'enseignement primaire. - L. 10 juill. 1998 portant approbation de la Convention du 31 octobre 1997 entre le Gouvernement, d'une part, et l'Archevêché, d'autre part, portant refixation des cadres du culte catholique et réglant certaines matières connexes.

5. L. 10 juill. 1998 portant approbation de la Convention du 31 octobre 1997 entre le Gouvernement, d'une part, et l'Église orthodoxe hellénique du Luxembourg, d'autre part. - L. 10 juill. 1998 portant approbation de la Convention du 31 octobre 1997 entre le Gouvernement, d'une part, et l'Église protestante du Luxembourg, d'autre part. - L. 10 juill. 1998 portant approbation de la Convention du 31 octobre 1997 entre le Gouvernement, d'une part, et les communautés israélites du Luxembourg, d'autre part. 
du Luxembourg et par l'extension aux orthodoxes roumains et serbes de la convention conclue avec l'Église hellénique en $1997^{6}$.

Le contenu de ces conventions est articulé autour de trois principes: une relative liberté d'organisation des cultes, l'organisation des institutions cultuelles dans le cadre d'un statut de droit public et le principe de la rémunération des ministres du culte par l'État.

À l'issue des élections législatives d'octobre 2013, une coalition majoritaire rassemblant les libéraux, les socialistes et les Verts a décidé de mettre en œuvre un programme de séparation de l'État et des religions ${ }^{7}$ assorti d'une réforme de la Constitution. Or, une révision constitutionnelle suppose une majorité des deux tiers des soixante députés, alors que le parti chrétien social, proche de l'Église catholique, rassemblait à lui seul vingt-trois élus. La sévérité du projet initial a finalement été atténuée par une négociation entre les différentes fractions, qui a permis la signature par l'État du Grand-duché de nouvelles conventions avec l'Église catholique et les autres cultes conventionnés (protestants, anglicans, orthodoxes, juifs, et musulmans qui s'ajoutent alors à la liste). Il n'est pas exclu que le Parlement, lors de la future discussion des lois d'approbation, refuse d'approuver ou censure certaines dispositions, ou que certaines d'entre elles soient portées devant une juridiction. Par ailleurs, les articles 22 et 106 de la Constitution devraient être remplacés par un nouvel article disposant qu'« en matière religieuse et idéologique, l'État respecte, en vertu du principe de séparation, les principes de neutralité et d'impartialité. La loi règle les relations entre l'État et les communautés religieuses ainsi que leur reconnaissance. Dans les limites et formes fixées par la loi, des conventions à approuver par la Chambre des députés peuvent préciser les relations entre l'État et les communautés religieuses reconnues $»^{8}$.

Les conventions signées le 26 janvier 2015 par les ministres concernés (respectivement Premier ministre, ministre de l'Éducation nationale et ministre

6. L. 11 juin 2004 autorisant l'État à prendre en charge les traitements et pensions des ministres du culte de l'Église anglicane du Luxembourg et conférant la personnalité juridique de droit public à ladite Église. - L. 11 juin 2004 autorisant l'État à prendre en charge les traitements et pensions des ministres du culte des Églises orthodoxes roumaine et serbe du Luxembourg et conférant la personnalité juridique de droit public auxdites Églises.

7. La cérémonie officielle du Te Deum de la fête nationale sur invitation de l'archevêque a été, dès 2014, remplacée par une manifestation civile, une cérémonie religieuse se tenant à la cathédrale l'après-midi.

8. Ce projet d'article a été approuvé à l'unanimité des membres de la Commission des institutions et de la révision constitutionnelle de la Chambre des députés. 
de l'Intérieur) et les représentants des cultes ${ }^{9}$ pour une durée de vingt ans précisent dans leurs préambules que les communautés religieuses bénéficiant de conventions ${ }^{10}$ " professent une religion reconnue au niveau mondial, sont bien établies au Luxembourg et y sont appuyées par une communauté suffisamment nombreuse ». Il est par ailleurs explicitement indiqué que les cultes signataires garantissent « le respect des droits et libertés constitutionnels, de l'ordre public et des valeurs démocratiques, la promotion des droits de l'homme et de l'égalité de traitement ainsi que de l'égalité entre hommes et femmes ». En outre, en contrepartie de la subvention versée par le gouvernement, " les cultes prêtent assistance à toute personne qui en formule la demande ». Conformément aux articles 2 des conventions, les communautés religieuses s'engagent «à écarter de l'organisation de la communauté tout membre qui agit ou qui appelle à agir en violation de ces principes ». Dans un tel cas, son subventionnement pourrait être supprimé. Les relations entre les communautés religieuses et le Grand-duché du Luxembourg sont ainsi soumises au principe du do ut des : l'octroi de soutiens symboliques et matériels des pouvoirs publics est subordonné au respect des valeurs communes et à la prise en charge des besoins spirituels.

Ces conventions sont chacune divisées en trois parties : dispositions communes à l'ensemble des cultes signataires; dispositions spécifiques à chaque culte et une troisième partie consacrée à la durée des conventions et aux dispositions abrogatoires. Les dispositions communes s'appliquent essentiellement à l'organisation et au fonctionnement des communautés religieuses. La liberté d'organisation est garantie, mais est limitée par le devoir pour les cultes de prendre en charge spirituellement les personnes qui en font la demande et par l'obligation de soumettre à l'approbation du gouvernement la désignation des chefs de culte.

Chaque communauté religieuse conventionnée est par ailleurs tenue d'indiquer aux autorités publiques un organe représentatif national auquel sera versée la subvention annuelle. Elle communique par ailleurs au ministre

9. L'Église catholique du Luxembourg, la communauté israélite du Luxembourg, l'Église protestante du Luxembourg résultant de la fusion de deux communautés protestantes conventionnées, la communauté musulmane du Luxembourg qui fait désormais partie du cercle des cultes conventionnés, l'Église anglicane du Luxembourg et l'Église orthodoxe du Luxembourg. L'Église catholique a en sus conclu deux conventions spécifiques. La première s'applique à l'enseignement religieux et au Grand séminaire, la seconde aux fabriques d'église.

10. Le terme de culte reconnu, remplacé par culte statutaire en droit local alsacien-mosellan, n'est plus repris dans la terminologie juridique luxembourgeoise. Il est encore utilisé en Belgique et en Autriche (anerkannte Religionsgemeinschaften). 
de la Justice le nom de son chef de culte, qui la représentera dans ses rapports avec le gouvernement. Les cultes conventionnés conserveront probablement un statut de droit public pour des raisons d'opportunité, cet aspect devant être précisé par les lois de publication des accords.

Le budget public qui était affecté à la rémunération des ministres du culte sera progressivement réduit de $70 \%$. Il passera d'ici vingt ans de 24,66 millions d'euros en 2015 à 8,375 millions d'euros pour l'ensemble des cultes conventionnés, soit de 23,73 millions d'euros à 6,75 millions d'euros pour l'Église catholique, durement touchée par cette réduction. L'Église protestante percevra $450000 €(635252 €$ en 2012), le culte israélite $315000 €(397973 €$ en 2012), l'Église orthodoxe $285000 €(261203 €$ en 2012) et l'Église anglicane $125000 €(151037 €$ en 2012). Le culte musulman, qui ne faisait jusqu'alors pas partie des cultes conventionnés est, à partir de 2015, subventionné à hauteur de $450000 €$ en application de l'article 31 de la convention. Ces sommes ont été fixées sur la base du nombre de membres de chaque culte, estimé d'un commun accord entre le culte concerné et les autorités étatiques.

Depuis le 26 janvier 2015, les communautés religieuses acceptent de ne plus recruter leurs collaborateurs sur le budget de l'État. Ils sont « engagés sous un régime de droit privé » (art. 4 des conventions). L'article 5 garantit toutefois aux ministres du culte engagés avant l'entrée en vigueur de la nouvelle convention le maintien des droits au traitement et à la pension fixés au moment de leur recrutement. Ces personnels sont cependant invités à faire valoir leur droit à pension à l'âge de soixante-cinq ans au plus tard, ce qui entraînera, notamment pour le culte catholique, une réduction rapide de la ligne budgétaire affectée aux personnels cultuels. Dès qu'elle sera inférieure à la subvention fixée par la convention de 2015, le gouvernement versera au culte concerné la différence puis, suite au départ à la retraite de tous les ministres du culte rémunérés par l'État, la totalité de la subvention.

Deux conventions spécifiques s'appliquant à l'enseignement religieux et à la gestion du temporel des paroisses ont été passées avec l'Église catholique. Le décret du 30 décembre 1809 concernant les fabriques des églises qui détaille les règles d'organisation, de fonctionnement et de financement de ces établissements, ainsi que le décret du 30 septembre 1807 augmentant le nombre de succursales seront abrogés à dater du $1^{\text {er }}$ avril $2017^{11}$. Les fabriques d'église seront remplacées par un Fonds de gestion des édifices religieux du culte catholique appelé le «Fonds », créé par voie législative. Cette personne

11. Convention concernant la nouvelle organisation des conseils de fabrique, art. $1^{\text {er }}$. 
juridique pourra recueillir des dons et des legs bénéficiant d'exonérations fiscales. Le Fonds aura notamment pour objectif « de fournir aux frais nécessaires du culte ». Il sera en charge du financement du culte paroissial et de l'entretien des bâtiments. Ce fonds, géré par un conseil d'administration dont les membres seront nommés par l'archevêque de Luxembourg, sera destinataire de tous les avoirs des fabriques d'église. La réforme inscrite dans la convention est sévère pour l'Église catholique dont le Fonds doit prendre en charge à partir de 2017 la totalité du temporel du culte local : logement des curés et desservants, frais de culte, entretien des églises et des presbytères, et cela grâce à la générosité des fidèles et au patrimoine transféré au Fonds par les fabriques. L'intervention des communes est prohibée par la convention.

La formation dispensée et la recherche menée au Grand séminaire catholique de Luxembourg auxquelles participeront les autres cultes conventionnés, seront subventionnées par l'État à hauteur de $600000 €$. Une convention entre le ministère de l'Enseignement supérieur et de la Recherche et le Grand séminaire précisant les engagements des deux parties sera conclue ultérieurement.

L'enseignement religieux dans les écoles publiques à destination des seuls élèves catholiques était organisé par l'administration, de concert avec l'archevêché qui fixait le contenu des cours et proposait pour nomination les enseignants du primaire et du secondaire. Il sera remplacé dès la rentrée 2016 par un cours commun à tous les élèves et figurant parmi les matières obligatoires, intitulé "éducation aux valeurs », dans le respect des procédures en vigueur au ministère de l'Éducation nationale : définition des programmes par la Commission nationale des programmes et validation par le ministre, qui s'engage cependant à associer à la réflexion les représentants de la société civile et le Conseil des cultes conventionnés, créé il y a quelques années comme interlocuteur de l'État du Grand-duché pour tout ce qui concerne le financement public et l'organisation des cultes. Le cours sera dispensé par les actuels professeurs de religion catholique dans le secondaire, qui seront remplacés progressivement, suite à leur départ à la retraite ou à leur reconversion, par des « enseignants spécialisés ». 